\title{
Laboratory analysis of organic acids, 2018 update: a technical standard of the American College of Medical Genetics and Genomics (ACMG)
}

\author{
Renata C. Gallagher MD, PhD ${ }^{1}$, Laura Pollard, $\mathrm{PhD}^{2}$, Anna I. Scott, PhD ${ }^{3,4}$, Suzette Huguenin, $\mathrm{PhD}^{5}$, \\ Stephen Goodman, MD ${ }^{6}$, Qin Sun, PhD 7 ; on behalf of the ACMG Biochemical Genetics \\ Subcommittee of the Laboratory Quality Assurance Committee
}

\begin{abstract}
Disclaimer: This laboratory standard is designed primarily as an educational resource for clinical laboratory geneticists to help them provide quality clinical laboratory genetic services. Adherence to this standard is voluntary and does not necessarily assure a successful medical outcome. This standard should not be considered inclusive of all proper procedures and tests or exclusive of other procedures and tests that are reasonably directed to obtaining the same results. In determining the propriety of any specific procedure or test, the clinical laboratory geneticist should apply his or her own professional judgment to the specific circumstances presented by the individual patient or specimen.

Clinical laboratory geneticists are encouraged to document in the patient's record the rationale for the use of a particular procedure or test, whether or not it is in conformance with this standard. They also are advised to take notice of the date any articular standard was adopted, and to consider other relevant medical and scientific information that becomes available after that date. It also would be prudent to consider whether intellectual property interests may restrict the performance of certain tests and other procedures.
\end{abstract}

Organic acid analysis detects accumulation of organic acids in urine and other body fluids and is a crucial first-tier laboratory test for a broad spectrum of inborn errors of metabolism. It is also frequently ordered as follow-up for a positive newborn screen result, as recommended by American College of Medical Genetics and Genomics newborn screening ACTion sheets and algorithms. The typical assay is performed by gas chromatography-mass spectrometry. These technical standards were developed to provide guidance for laboratory practices in organic acid analysis, interpretation, and reporting. In addition, new diagnostic biomarkers for recently discovered organic acidurias have been added.

Genet Med advance online publication 15 March 2018

Key Words: clinical genetic testing; gas chromatography-mass spectrometry; organic acid; organic aciduria; technical standards

\section{INTRODUCTION}

Organic acids are analyzed in patients suspected of having a broad range of metabolic disorders including inborn errors of amino acid, fatty acid, carbohydrate, neurotransmitter, vitamin, sterol, mitochondrial energy, and purine and pyrimidine metabolism. Organic acids are non-amine-containing, water-soluble compounds with one or more carboxyl groups as well as other functional groups (-keto, -hydroxyl), and are intermediates of a large number of biochemical pathways. Organic acid analysis is performed to identify abnormal accumulation of one or more compounds resulting from an enzymatic or transporter deficiency. Exogenous compounds are also detected, and these may be relevant to the clinical presentation. The kidneys efficiently excrete organic acids, both endogenous and exogenous, therefore urine is the preferred specimen type.

Organic acids are extracted from urine using organic solvents or ion exchange methods, and their presence and abundance are determined by gas chromatography-mass spectrometry (GC-MS). Over 500 organic acids have been identified in urine. ${ }^{1}$ Both qualitative and quantitative analyses are appropriate for the detection of organic acidurias, with careful quality assessment and control. Diagnostic compounds are often significantly elevated in classic organic acidurias, so a diagnosis is possible without quantitative methods. It is important to note that for some disorders diagnostic compounds may be normal or near-normal in clinically well patients. ${ }^{1,2}$ Care must be taken to examine the

\footnotetext{
${ }^{1}$ Department of Pediatrics, University of California, San Francisco, San Francisco, California, USA; ${ }^{2}$ Greenwood Genetic Center, Greenwood, South Carolina, USA; ${ }^{3}$ Department of Pathology, Stanford University Medical Center, Stanford, California, USA; ${ }^{4}$ Department of Pathology, Seattle Children's Hospital, Seattle, Washington, USA; ${ }^{5}$ Laboratory Corporation of America Holdings (LabCorp), Research Triangle Park, North Carolina, USA; ${ }^{6}$ Department of Pediatrics, University of Colorado School of Medicine, Aurora, Colorado, USA; ${ }^{7}$ Department of Molecular and Human Genetics, Baylor College of Medicine, Houston, Texas, USA. Correspondence: Qin Sun (qsun@bcm.edu) The Board of Directors of the American College of Medical Genetics and Genomics approved this technical laboratory standard on 22 January 2018. 
chromatogram for clinically significant compounds that may be present in small amounts or obscured by coelution with other compounds; proficiency testing programs have reported missed diagnoses from poor detection of low-abundance pathognomonic compounds. ${ }^{3}$ Quantitative analyses are particularly useful for therapeutic monitoring of previously diagnosed patients. ${ }^{1-5}$

Specific organic acids (e.g., methylmalonic acid, glutaric acid, 3-hydroxyglutaric acid, orotic acid, and succinylacetone) can be determined in blood or urine using other techniques that utilize stable isotope dilution and GC-MS or liquid chromatography-tandem mass spectrometry. These targeted analyses should not replace a more comprehensive organic acid screen in the initial laboratory evaluation of undiagnosed cases. This technical standard focuses on untargeted urine organic acid analyses by GC-MS.

\section{METHODS}

The laboratory technical standard was informed by a review of the literature, including any current guidelines, and expert opinion. Resources consulted included PubMed (search terms: organic acidemias, organic acid analysis methods, organic acid extraction methods), the American College of Medical Genetics and Genomics (ACMG) Standards and Guidelines for Clinical Genetics Laboratories, Clinical and Laboratory Standards Institute guidelines, CLIA regulations, and the Centers for Disease Control and Prevention Morbidity and Mortality Weekly Report on Good Laboratory Practices for Biochemical Genetics Testing and Newborn Screening for Inherited Metabolic Disorders. When the literature provided conflicting evidence about a topic or when there was insufficient evidence, the authors used expert opinion to inform the recommendations. Expert opinion included the coauthors of the document, members of the Biochemical Genetics Subcommittee of the Lab Quality Assurance Committee, as well as any experts consulted outside the workgroup and acknowledged in this document. Any conflicts of interest for workgroup members or consultants are listed. The ACMG Laboratory Quality Assurance Committee reviewed the document providing further input on the content, and a final draft was delivered to the ACMG Board of Directors for review and approval to send out for member comment. The final draft of the document was posted on the ACMG website and an e-mail link was sent to ACMG members inviting all to provide comment. All members' comments were assessed by the authors and additional evidence was included and our recommendations were amended as deemed appropriate. Member comments and author responses were reviewed by a representative of the Laboratory Quality Assurance Committee and the ACMG Board. The final document was approved by the ACMG Board of Directors. This updated technical standard replaces the previous version in Section F: Clinical Biochemical Genetics, American College of Medical Genetics and Genomics Standards and Guidelines for Clinical Genetics
Laboratories (2008 Edition, Revised February 2007), section F7.7. ${ }^{6}$

\section{BACKGROUND}

\subsection{Overview of diseases identified through urine organic acid analysis}

Organic acidurias (also known as organic acidemias) are a biochemically heterogeneous group of inborn errors of metabolism. They are characterized by the accumulation of metabolites that are not present, or are only present in small amounts, under normal physiological conditions. Abnormalities detected by organic acid analysis include pathologically elevated quantities of normal metabolites, and compounds produced by alternative biochemical pathways (e.g., succinylacetone). The possible, or likely, diagnosis of a specific organic aciduria, or other disorder, relies on pattern recognition and the determination of the presence, or absence, of expected compounds. An identified defect in organic acid metabolism can be due to an enzyme deficiency; a disorder of cofactor biosynthesis, recycling, or modification; a transporter defect; or nutritional deficiency. Disorders that may be identified or suggested through abnormal urine organic acid analysis include the classic organic acidemias due to inborn errors of amino acid metabolism (isovaleric acidemia [MIM 243500], methylmalonic acidemia[s], propionic acidemia [MIM 606054], glutaric acidemia type I [MIM 231670]), as well as other amino acid disorders (phenylketonuria [MIM 261600], tyrosinemia type I [MIM 276700], alkaptonuria [MIM 203500], 3-methylglutaconic aciduria type I [MIM 250950], maple syrup urine disease [MIM 248600]). Abnormal urine organic acids may also be consistent with disorders of the urea cycle (suggested by elevated orotic acid), fatty acid oxidation (short-chain acyl-CoA dehydrogenase deficiency [MIM 201470], medium-chain acyl-CoA dehydrogenase deficiency [MIM 201450], multiple acyl-CoA dehydrogenase deficiency [MIM 231680]), energy metabolism (pyruvate dehydrogenase deficiency, fumarase deficiency [MIM 606812], SUCLA2 deficiency [MIM 603921]), purine and pyrimidine metabolism (uridine monophosphate synthetase deficiency [MIM 613891], dihydropyrimidine dehydrogenase deficiency [MIM 274270]), neurotransmission (aromatic Lamino acid decarboxylase deficiency [MIM 608643]), and others (ethylmalonic encephalopathy pMIM 602473), Canavan disease [MIM 271900], glutathione synthetase deficiency [MIM 266130], glycerol kinase deficiency [MIM 307030], primary hyperoxaluria type I [MIM 259900] and type II $\left[\right.$ MIM 260000]). ${ }^{7-9}$ Clinical indications for this testing are, therefore, diverse and include neonatal or late-onset acute illness associated with hyperammonemia, hypoglycemia, and/ or ketolactic acidosis; neurologic abnormalities including seizures, ataxia, hypotonia, lethargy, coma, developmental delay, or unexplained intellectual disability; failure to thrive; pancreatitis; unexplained metabolic acidosis; unusual odor; macrocephaly; and liver failure. ${ }^{10,11}$ Some symptoms, including lethargy and acidosis, can be due to exogenous intoxication, and organic acid analysis may aid in diagnosis 
(e.g., ethylene glycol poisoning, ibuprofen overdose, and $\gamma$ hydroxybutyric acid intoxication as distinct from succinic semialdehyde dehydrogenase deficiency [MIM 271980]).

\subsection{Incidence}

The incidence of individual classical inborn errors of organic acid metabolism varies from 1 in 10,000 to 1 in 1,000,000 live births. Collectively, their incidence has been suggested to be approximately 1 in 10,000-35,000 live births. ${ }^{8,12,13}$ This estimate, however, does not include other inborn errors of metabolism (i.e., amino acid disorders, urea cycle disorders, congenital lactic acidemias) for which diagnosis may also be suggested by organic acid analysis. All possible disease entities included, the incidence of conditions for which informative organic acid profiles can be detected in urine is likely to be higher than recognized. As a group, these defects are likely underdiagnosed.

\subsection{Mode of inheritance}

Most organic acidemias, and other inborn errors of metabolism identified through organic acid analysis, are inherited as autosomal recessive traits. X-linked disorders that may be identified by urine organic acid analysis include the amino acid and mitochondrial energy metabolism disorder, 2methyl-3-hydroxybutyryl-CoA dehydrogenase deficiency (MIM 300438); the urea cycle disorder, ornithine transcarbamylase deficiency (MIM 311250); the energy metabolism disorder, pyruvate dehydrogenase E1- $\alpha$ deficiency (MIM 312170); and glycerol kinase deficiency (MIM 307030). The identification of an affected individual has important implications for other family members, who may also require urine organic acid analysis and additional genetic testing.

\section{PREANALYTICAL REQUIREMENTS}

\subsection{Specimen requirements}

Organic acids are preferably analyzed in urine. Urine specimens are normalized to a fixed equivalent of creatinine to allow determination of relative concentration. Sample volume required for testing depends on the patient's hydration status. The minimal sample volume will also depend on specifics of the organic extraction; typically $1-5 \mathrm{ml}$ of specimen is recommended.

\subsection{Conditions of sample collection, shipping, handling, and storage}

Urine is ideally collected at the time of acute illness. If the patient is decompensated the urine should be obtained at the time of decompensation, as diagnostic metabolites are likely to be highest in this state. ${ }^{1,14}$ If the patient is not ill, a random urine sample is appropriate. ${ }^{14,15}$ Urine should be collected in a clean container without preservatives, frozen as soon as possible, and stored frozen until analyzed. Samples sent to the laboratory from outside referral sources should be shipped on dry ice via overnight courier. Urine samples are stable for most organic acids for several years if stored at $-20{ }^{\circ} \mathrm{C}$ or lower. ${ }^{1}$

\subsection{Exogenous variables}

Diet, medication, and clinical state may contribute to an abnormal organic acid profile. ${ }^{1}$ Dietary factors include total parenteral nutrition (isolated $\mathrm{N}$-acetyltyrosine), gelatin (isolated adipic acid), medium-chain triglyceride oil supplementation (dicarboxylic aciduria), infant formula (dicarboxylic aciduria due to medium-chain triglyceride and other artifacts), and the ketogenic diet (ketoaciduria). Medications such as valproate, levetiracetam, aspirin, benzoic acid, ibuprofen, acetaminophen, and many others can be the source of a wide variety of exogenous peaks. ${ }^{16}$ Treatment with certain medications may also be associated with increased production and excretion of organic acids with diagnostic relevance. For example, valproate treatment inhibits 3methylcrotonyl-CoA carboxylase and results in elevated urinary excretion of 3-hydroxyisovaleric acid, ${ }^{17}$ a marker of biotinidase deficiency and other disorders. Pyroglutamic acid (5-oxoproline) elevation, a marker of glutathione synthetase deficiency, may also occur secondary to treatment with acetaminophen, vigabatrin, and other medications. ${ }^{18,19}$ Clinical information may be required to determine if these are relevant to the clinical presentation (e.g., ibuprofen overdose). Sample contamination can also lead to abnormal findings. For example, lubricants or soaps can result in elevations of glycerol (also elevated in glycerol kinase deficiency), palmitic acid, and stearic acid. Mild elevations of methylmalonic acid can be secondary to intestinal bacteria metabolism or nutritional status (low infant or maternal B12 or folate). Compounds of bacterial origin can be enhanced with prolonged sample storage at ambient temperature. ${ }^{20}$ Clinical history, diet, and medication should be considered to aid the interpretation of the chromatographic findings.

\subsection{Clinical indications for testing}

Urine organic acid analysis should be performed for patients suspected of having a wide range of disorders based on clinical symptoms and/or other laboratory abnormalities. Clinical symptoms are varied and often nonspecific, as described above in section 3.1, and can occur for the first time at any age from the neonatal period to adulthood. Certain clinical findings may be associated with particular disorders, such as macrocephaly in Canavan disease (MIM 271900) and glutaric acidemia type I (MIM 231670), and neonatal liver disease in tyrosinemia type I (MIM 276700). A family history of a previously affected sibling or an abnormal newborn screen result may suggest a specific inborn error of metabolism.

Organic acid analysis is required as part of newborn screening confirmatory testing when the differential diagnosis includes an organic aciduria(s) as well as other disorders. Follow-up newborn screening recommended testing algorithms and ACTion (ACT) sheets are available at the ACMG website. ${ }^{21,22}$ Newborn screening platforms, analytes, analyte cutoffs, protocols, and algorithms differ widely. A "normal" newborn screen, one in which none of the screened analytes were outside of the established high and low cutoffs, should 
not be considered diagnostic testing for any disorder screened by that program; diagnostic testing, including organic acid analysis and other testing, should be ordered if there is clinical suspicion for a particular disorder(s). There is worldwide interest in continued assessment and optimization of newborn screening, due to its benefit in early diagnosis of organic acidurias and other conditions and programs may change platforms and adjust cutoffs over time. ${ }^{23-27}$

For optimal interpretation, the laboratory should be made aware of the clinical indication for testing, the clinical state of the patient at the time of the testing, as well as concurrent medications and diet. The laboratory should have contact information for the ordering physician to aid interpretation, if needed, and to communicate urgent and emergent results. Depending on the clinical situation, and in a diagnostic evaluation, organic acid analysis should be ordered with analyses of plasma amino acids, acylcarnitine profile, and carnitine (free and total). Ideally, these tests should all be performed by the same laboratory, and the results integrated into an overall interpretation.

\section{METHOD ESTABLISHMENT AND VALIDATION}

\subsection{Qualitative versus quantitative analysis}

Urine organic analysis can be performed as a qualitative or quantitative test and both methods are effective approaches for establishing a diagnosis. Qualitative urine organic acid analysis reports contain written descriptions of the observed pattern with an overall interpretation. Quantitative urine organic analyses typically determine concentrations of selected metabolites through the use of numerous calibrators and internal standards. Qualitative and quantitative analyses share multiple assay parameters. The volume of urine analyzed generally contains a fixed amount of creatinine and, therefore, varies with patient hydration status. Abnormal metabolites are identified by both retention $\operatorname{time}^{28}$ and fragmentation pattern in the mass spectrometer. Typically, each laboratory develops a custom library of compounds that identify retention time and relative ion abundance based on instrument parameters such as flow rate, column type, column length, and elution ramp temperatures. Given the challenges of manual organic acid extraction, internal standards are necessary to ensure proper sample preparation (see section 5.2).

Quantitative analysis offers certain benefits and caveats. Some clinicians find quantitative reports helpful for monitoring known patients. In this case, clinicians should be aware that, in the absence of stable isotope dilution, or another robust quantitative method, quantitative results may not be portable between laboratories so therapy monitoring should be performed in one laboratory. ${ }^{1}$ For metabolites excreted in low levels among clinically normal individuals, quantitative methods, along with reference values, may be helpful to determine whether an analytical level is indicative of an inborn error of metabolism. However, pathological levels of certain metabolites, such as mevalonic acid, may be minimal, and below the limit of detection of some methods, and thus, more sensitive targeted assays are necessary with stable isotope dilution using selected ion monitoring. ${ }^{29,30}$

\subsection{Calibration and quantitation}

Urine organic acid analysis should be performed using at least one internal standard. The choice of internal standard varies between laboratories but it should be uniquely identifiable, not present in human specimens, and resolvable from other compounds by gas chromatography. The internal standard controls for extraction efficiency, and allows the direct comparison of metabolite concentrations in patient specimens to calibration standards. Commonly used internal standards include heptadecanoic acid, 2-phenylbutyric acid, ${ }^{31}$ tropic acid, pentadecanoic acid, ${ }^{15}$ 2-ketocaproic acid, ${ }^{32}$ p-chlorobenzoic acid, and dimethylmalonic acid. ${ }^{33} \mathrm{~A}$ second internal standard added immediately before derivatization may help assess instrument function; tetracosane is commonly used as a second standard.

Quantification of organic acid concentrations must be performed by comparison with a known reference or external calibration standard(s) whenever possible. A calibration curve is prepared for each analyte quantified during the procedure, covering the normal range and expected pathological values. The calibration curve should include several dilutions of the standard mix containing the organic acids quantified. To generate and update calibration curves, calibrators should be carried through the entire specimen preparation process. Usually, only a subset of organic acids is quantified, and most other organic acids are assessed qualitatively. Quantification could be performed using a single quantifying ion for each compound. The peak area of the compound quantifying ion is normalized to the peak area of the internal standard ion and sample volume, and then quantitation is obtained using the calibration curve slope. ${ }^{32,34}$ The internal standards used are typically nonisotopic compounds unless specific organic acids are targeted. The use of solvent standards (i.e., standards mixed directly in organic solvent and not carried through the full extraction process) is inappropriate because these do not adequately control for different extraction efficiencies. ${ }^{20}$ Quantitation without the use of a specific reference standard is discouraged. However, for compounds for which quantitative data is desired but no reference standard is available, the laboratory may compare peak response with that of a known quantity of internal standard to derive a relative concentration. In these cases, the metric should be clearly indicated on the report.

\subsection{Reference ranges}

Reference ranges for all reported quantified compounds should be established and periodically validated. Reference ranges should be normalized to the creatinine concentration. When literature-based ranges are used they must also be periodically verified. Age-specific ranges should be determined for compounds for which the concentration is known

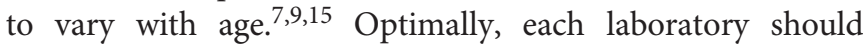
establish its own reference ranges based on its method. 


\subsection{Validation}

Validation of qualitative organic acid analysis utilizes urine samples from unaffected controls and patients with previously confirmed inborn errors of metabolism. The proper identification of expected abnormal metabolites in abnormal control specimens (and absence in unaffected control specimens) should be documented for as many inborn errors of metabolism as is reasonably possible. Proficiency testing programs are one source of confirmed abnormal samples. Specimen exchange between testing laboratories is also an effective means of increasing exposure to rare diseases, and of validating a new method or instrument.

For quantitative analysis, the laboratory should establish protocols to determine the linear range, analytical measurement range, and lower limit of quantification for all quantified compounds. Procedures should be in place for addressing any values that fall outside of these performance limits. Initial validation should include analysis of the precision and recoveries for each quantified analyte. Performance characteristics (e.g., linearity, analytical measurement range, accuracy, and precision) should be determined for all quantified analytes and verified at appropriate, regular intervals. ${ }^{14}$ Instrument calibration should be validated and verified at regular intervals established by the laboratory and as required by CLIA. Calibration verification should also be performed following any major service to the instrument, or before the use of a new lot of reagents.

\subsection{Testing personnel}

Appropriate training procedures and ongoing competency requirements for laboratory personnel performing organic acid analysis must be established and documented. Organic acid analysis is considered high-complexity testing, so personnel should, at minimum, satisfy CLIA requirements. Specific training in peak identification and interpretation may be necessary.

\section{ANALYSIS OF ORGANIC ACIDS \\ 6.1 Extraction of organic acids}

The volume of urine required for organic acid analysis is dependent on its creatinine concentration. The sample volume should be adjusted to reach a set creatinine amount, and dilution of concentrated samples performed as necessary. A volume of urine equivalent to $0.25 \mathrm{mg}(2.2 \mu \mathrm{mol})$ of creatinine is typically used; various protocols call for 0.1 to $0.4 \mathrm{mg}$ of creatinine. One or more internal standards is added to the specimen (see section 5.2), followed by the addition of an acid such as hydrochloric acid. Addition of sodium chloride or urease prior to extraction can reduce the amount of urea, which may interfere with other compounds. 3,35 Urease treatment nonextraction methods are reported to increase detection of glycerol and glycerol-3-phosphate, aiding in the diagnosis of fructose-1,6-bisphosphatase deficiency, and are capable of screening carbohydrates and amino acids in addition to organic acids. ${ }^{35,36}$
Extraction of organic acids from acidified urine ( $\mathrm{pH} \mathrm{1-2)} \mathrm{is}$ performed using organic solvents or ion-exchange solid-phase extraction. Traditional protocols use diethyl ether and/or ethyl acetate as the preferred organic solvents. ${ }^{15,33}$ Alternatively, tetrahydrofuran shows higher sensitivities for polar compounds than ethyl acetate. ${ }^{37}$ Solid phase extraction with strong anion exchange cartridges is sometimes favored to improve extraction of more polar compounds without urine acidification, though it can have reduced extraction efficiency of less polar, pathologically relevant metabolites. ${ }^{38,39}$ Less polar compounds such as orotic acid, succinylacetone, and uracil may be poorly extracted depending on the specific method used. A targeted quantitative analysis may be warranted to query these specific metabolites. Alternatively, prior to acidification and extraction, an oximation step can stabilize and improve detection of keto acids such as pyruvic acid, $\alpha$-ketoglutaric acid, succinylacetone, and certain intermediates of branched-chain amino acid metabolism: 2ketoisocaproic acid, 2-keto-3-methylvaleric acid, and 2ketoisovaleric acid. Oximation may be performed routinely or under select circumstances, such as the clinical suspicion of maple syrup urine disease (MIM 248600) or tyrosinemia type I (MIM 276700).

\subsection{Derivatization}

Following extraction, an additional standard, often tetracosane, can be added to the specimen. Organic extracts are then evaporated to dryness under nitrogen and derivatized as described below. Incomplete dryness can reduce derivatization efficiency.

Trimethylsilyl derivatives of organic acids in dried urine extracts are formed by the addition of $\mathrm{N}, \mathrm{O}$-bis (trimethylsilyl) trifluoroacetamide with $1 \%$ trimethylchlorosilane (BSTFA/ TMCS) or similar commercial reagents. In a typical derivatization procedure, dried extracts are mixed with BSTFA/TMCS and incubated at $65-90{ }^{\circ} \mathrm{C}$ for $10-30$ minutes. Alkane standards of known carbon chain length may be added to monitor chromatographic separation.

\subsection{Gas chromatography-mass spectrometry instrumentation}

Several instrument configurations are commercially available that separate compounds and enable identification by chromatographic retention time and mass spectra. Separation is most often carried out on a capillary column, and mass spectra obtained from either a quadrupole filter or ion trap mass spectrometer using electron impact ionization. Fixed electron impact energy is applied to generate reproducible and portable spectra. A small amount of sample $(1-3 \mu \mathrm{l})$ is typically injected. Application of a split inlet mode helps to avoid column overload with a split ratio of 1:15-70. Mass spectral data are typically collected in full scan mode from $\mathrm{m} /$ z 50 to 550 to generate total ion current chromatograms. Selected ion monitoring may be used by methods that target specific compounds. 


\subsection{Chromatogram analysis}

Total ion current chromatograms are analyzed by manual interrogation of the spectral data. Compound identification is critical to the diagnosis of genetic disorders associated with abnormal organic acid excretion and relies on the evaluation of both their mass spectra and retention time. A computer library of mass spectra of known compounds is typically used to confirm the identity of metabolite peaks. A limited number of commercial libraries (e.g., the National Institute of Standards and Technology and Wiley) containing mass spectra of trimethylsilyl derivatives of physiologic and drugrelated compounds are available for the identification of compounds and are compatible with most GC-MS manufacturer software packages. These commercial reference libraries may not contain all compounds of interest to biochemical genetics laboratories. The common, nonsystematic chemical names may not be available in these reference libraries. Laboratories are encouraged to modify commercial reference libraries and develop in-house libraries on their own equipment, using pure organic acid standards and/or patientderived peaks (once positive identification of a compound has been established). A minimal library in a biochemical genetics laboratory should contain all of the compounds in the expanded newborn screening follow-up testing guideline by National Academy of Clinical Biochemistry and organic acid master list in the College of American Pathologists (CAP) Biochemical Genetics Survey. ${ }^{1,28}$ It is good laboratory practice to continuously update the analytical library when new diagnostic compounds are reported in the literature.

The methods and criteria by which peaks are identified must be documented in the laboratory. Although instrument software can generate analyte match reports according to preset criteria, manual review of peaks and spectra is necessary to achieve accurate peak identification. ${ }^{1}$ Examination of chromatographic retention times and/or relative retention times to an internal standard is also necessary, particularly for the correct identification of isomers or spectrally similar compounds. ${ }^{9}$ Particular attention should be paid to regions of the chromatogram in which complete or partial overlap of a clinically relevant peak with that of another compound could hinder identification. Possible critical organic acid overlaps include 3-hydroxybutyric acid/ 3-hydroxyisobutyric acid, urea/4-hydroxybutyric acid, hexanoylglycine/4-hydroxyphenylacetic acid, ethylmalonic acid/ phosphoric acid, 3-methylglutaconic acid/3-hydroxyadipic lactones, tiglylglycine/3-methylcrotonylglycine, orotic acid/ cis-aconitic acid, and others. ${ }^{1,15}$ Modification of analytical system parameters such as column type, elution temperature ramp, and run time may resolve some of these coeluting compounds. Examinations of postrun extraction ion chromatograms and subtracted spectra are often helpful to clarify the composition of apparently complex peaks. Care must be taken to examine each chromatogram for the presence of peaks that, when present even in small amounts, could be clinically significant (e.g., hexanoylglycine, suberylglycine, succinylacetone, 2-methylcitric acid, and orotic acid).

\subsection{Quality control}

The mass spectrometer should be tuned prior to each batch of patient samples analyzed, as described by the instrument manufacturer and required by the CAP. Procedures should be developed and documented for the routine evaluation of system performance, as well as the detection and evaluation of potential carryover effects. A quality control (QC) program based on the analysis of normal and abnormal controls should be implemented and performed with every batch of patient specimens. Peak areas of internal standards should be monitored for extraction and derivatization efficiency in all patient and QC specimens. Thresholds for acceptance or rejection of QC data, and remedial actions in the event of a QC failure, should be established and documented by the laboratory. QC data should be regularly monitored for overall trends that may affect test performance, and problems should be documented and remediated as appropriate. The use of Westgard rules for clinical specimen analysis further specifies the parameters for quality patient diagnosis and reporting. ${ }^{40}$

\subsection{Proficiency testing}

An ongoing proficiency testing program for organic acid analysis must be implemented and documented. A biannual proficiency testing program that evaluates both analytical and interpretive/diagnostic proficiency is offered by the CAP and supervised by the joint CAP/ACMG Genetic Biochemical and Molecular Genetic Resource Committee. ${ }^{28}$ An excellent program for both qualitative and quantitative organic acid analysis is also offered by the European Research Network for evaluation and improvement of screening, Diagnosis and treatment of Inherited disorders of Metabolism (ERNDIM; http://www.erndimqa.nl)..$^{2,5,41}$ In addition to the official CAP and ERNDIM testing, sample exchange between laboratories and sharing of unusual data are valuable educational resources.

\section{TEST INTERPRETATION AND REPORTING \\ 7.1 Interpretation}

Urine organic acid analysis may be the most complex test performed in many biochemical genetic laboratories. Interpretation of urine organic acid results should be performed by an American Board of Medical Genetics and Genomics or other relevant medical board-certified laboratory director. Clinically meaningful interpretation of organic acid results should be based on the overall pattern of metabolites present in abnormal quantities, rather than on individual abnormalities. The diagnostic sensitivity of organic acid analysis under acute and asymptomatic clinical states may vary considerably. Although certain diagnoses can be confidently suggested based on an organic acid profile, there are limitations of this test. Informative profiles may not always be detected in disorders in which the excretion of diagnostic metabolites is a reflection of the residual activity of the defective enzyme, the dietary load of precursors, and the anabolic/catabolic status of the patient. For example, some individuals with glutaric acidemia type I (MIM 231670) are considered to be "low 
Table 1 Recently described inborn errors of metabolism with abnormalities in urine organic acid analysis

Disorder

Aminoacylase 1 deficiency, MIM 609924 (ref. 44)

Aromatic L-amino acid decarboxylase deficiency (primary or due to abnormal B6 level or metabolism), MIM 608643 (refs. 45,46) Combined malonic and methylmalonic aciduria, MIM 614265 (ref. 47)

3-OH-Isobutyryl-CoA hydrolase deficiency, MIM 250620 (ref. 48)

3-OH-3-Methylglutaryl-CoA synthase 2 deficiency, MIM 605911

(refs. 49,50)

Methylmalonyl-CoA epimerase deficiency, MIM 251120 (ref. 51)

Combined D-2 and L-2-hydroxyglutaric aciduria, MIM 615182 (ref. 52) D- and L-2-hydroxyglutaric acid

Cytosolic phosphoenolpyruvate carboxykinase deficiency, MIM

261680 (ref. 53)

Riboflavin transporter defect, MIM 211530 and MIM 211500 (ref. 54) Ethylmalonic acid, adipic acid, 2-hydroxyglutaric acid, suberic acid,

Short/Branched-chain acyl-CoA dehydrogenase deficiency, MIM

610006 (refs. 55,56)

Short-chain enoyl-CoA hydratase deficiency, MIM 616277 (ref. 48)

Succinate-COA ligase (SUCLA2 and SUCLG1) deficiencies, MIM

612073 and MIM 612224 (refs. 57,58)

Succinic semialdehyde dehydrogenase deficiency, MIM 271980

(ref. 59)

Xanthurenic aciduria, MIM 236800 (ref. 60) hexanoylglycine, and suberylglycine

$N$-acetyl amino acids

Vanilpyruvic acid, 3-O-methyldopa (3-methoxytyrosine), N-acetylvanilalanine, vanillactic acid

2,3-Dihydroxy-2-methylbutyric acid $^{a}$

3,5-Dihydroxyhexanoic 1,5 lactone, trans-5-hedroxyhex-2-enoate, 4-hydroxy-6methyl-2-pyrone, 5-hydroxy-3-ketohexanoate, 3,5-dihydroxyhexanoate

Ketones, methylmalonic acid, 3-hydroxypropionic acid, methylcitric acid, propionylglycine

Tricarboxylic acid cycle metabolites: fumaric acid, succinic acid, malic acid,

$\alpha$-ketoglutaric acid, adipic acid, 3-hydroxysebacic acid, and

2-Ethylhydracrylic acid, 2-methylbutyrylglycine

2,3-Dihydroxy-2-methylbutyric acid ${ }^{a}$

Methylmalonic acid, methylcitric acid, and others

4-hydroxybutyric acid, 3,4-dihydroxybutryric acid, dicarboxylic Aciduria, 4,5-dihydroxyhexanoic acid, and lactones (not present in $\gamma$-hydroxybutyric acid intoxication)

Xanthurenic acid

${ }^{a}$ Follow-up testing is indicated to look for urine acrylate conjugates, and molecular studies are recommended. ${ }^{48}$

excretors" in that urinary glutaric acid and 3-hydroxyglutaric acid levels may not demonstrate significant abnormalities. ${ }^{42}$ Communication with the ordering physician to better understand the clinical, medication, and nutritional history may help the laboratory director accurately interpret a complex chromatogram. Comprehensive tables are available in published literature and online to assist in interpretation of organic acid findings. ${ }^{7,9,19,43}$ A brief table of recently identified organic acidurias and their associated abnormal organic acids is provided here (Table $\mathbf{1}$ ).

A characteristic abnormal organic acid profile may not be sufficient to establish a definitive diagnosis. Other methods of higher specificity and sensitivity based on stable isotope labeled internal standards, selected ion monitoring, or chemical ionization can overcome the limitations of standard organic acid analysis for the investigation of nonacutely ill or low excretor patients (e.g., targeted methods for the determination of methylmalonic acid, 3-hydroxyglutaric acid, or succinylacetone). In some instances targeted quantification of specific metabolites in other physiological fluid (cerebrospinal fluid, amniotic fluid, plasma, or serum) may be appropriate to clarify an ambiguous urine finding. Confirmation by an independent method is recommended whenever practical, such as molecular analysis, associated analyte determination, or in vitro enzyme assay (blood cells, cultured cells, or tissue biopsy). Such follow-up testing is particularly helpful to clarify nonspecific organic acid elevations such as tricarboxylic aciduria suggestive of mitochondrial dysfunction, vanillactic acid suggestive of a neurotransmitter synthesis defect, or hypoglycemic ketonuria.

\subsection{Reporting}

According to a 10 -year review by ERNDIM, report quality and content varied greatly upon ambiguous findings in qualitative organic acid assay. ${ }^{5}$ Reports should be clear to a nonspecialist. Patient reports must contain appropriate patient and specimen information as contained in sections C2.4, 2.4.1, and 2.4.2 of the American College of Medical Genetics Standards and Guidelines for Clinical Genetics Laboratories (2008 Edition). ${ }^{6}$ Identification of all relevant compounds must be provided on the report, and quantity may be listed (as determined). Quantitative reports must include appropriate reference ranges (see section 5.3). The report may include a brief description of the method and a list of known analytes that are not well determined. When no clinically significant abnormalities are detected, an organic acid analysis can be reported and interpreted in qualitative terms only. When abnormal results are detected, the detailed interpretation should include an overview of the results and their potential significance, correlation with available clinical information, a differential diagnosis, recommendations for additional confirmatory or other follow-up studies, and 
contact information for the reporting laboratory. In the case of a new diagnosis the laboratory should attempt to reach the ordering physician to ensure the recommendations are communicated directly. If appropriate, referral of the patient to a metabolic center for further evaluation and management should also be recommended, as this is likely to optimize the patient outcome. ${ }^{5}$

\section{ACKNOWLEDGMENTS}

Members of the ACMG Biochemical Genetics Subcommittee and the Laboratory Quality Assurance Committee reviewed and endorsed the revised document. We thank Irene De Biase for assistance with section 5.2 .

\section{DISCLOSURE}

The following authors direct laboratories that perform urine organic acid analysis as a fee-for-service: L.P., A.I.S., S.H., S.G., and Q.S. The other authors declare no conflict of interest.

\section{REFERENCES}

1. Dennis J, Dietzen PR, Whitley RJ, et al. Follow-up testing for metabolic diseases identified by expanded newborn screening using tandem mass spectrometry. In: Bennett MJ, (ed). National Academy of Clinical Biochemistry, 2009. https://www.aacc.org/science-and-practice/practiceguidelines/expanded-newborn-screening.

2. Peters V, Garbade SF, Langhans CD, et al. Qualitative urinary organic acid analysis: methodological approaches and performance. J Inherit Metab Dis 2008;31:690-696.

3. Hoffmann G, Aramaki S, Blum-Hoffmann E, Nyhan WL \& Sweetman L. Quantitative analysis for organic acids in biological samples: batch isolation followed by gas chromatographic-mass spectrometric analysis. Clin Chem 1989;35:587-595.

4. Goodman SI \& Markey SP. Diagnosis of organic acidemias by gas chromatography-mass spectrometry. Lab Res Methods Biol Med 1981;6: $1-158$.

5. Peters V, Bonham JR, Hoffmann GF, Scott C \& Langhans CD. Qualitative urinary organic acid analysis: 10 years of quality assurance. J Inherit Metab Dis 2016;39:683-687.

6. American College of Medical Genetics and Genomics. Standards and guidelines for clinical genetics laboratories. Section F7.7. 2008 edition, revised. 2007. https://www.acmg.net/docs/Section_F_2008_revision.pdf.

7. Chalmers R \& Lawson AM. Organic Acids in Man: Analytical Chemistry, Biochemistry and Diagnosis of the Organic Acidurias. Chapman \& Hall: London, 1982.

8. Lehotay DC \& Clarke JT. Organic acidurias and related abnormalities. Crit Rev Clin Lab Sci 1995;32:377-429.

9. de Almeida I \& Duran M. Organic acids. In: Blau N, Duran M, Gibson KM, Dionisi-Visi C (eds). Physician's Guide to the Diagnosis, Treatment, and Follow-up of Inherited Metabolic Diseases. Springer: Heidelberg, Germany, 2014:761

10. Blau N, Duran M \& Blaskovics ME. Physician's Guide to the Laboratory Diagnosis of Metabolic Diseases. Chapman \& Hall Medical: London, 1996.

11. Rinaldo P, Hahn SH \& Matern D. Inborn errors of amino acid, organic acid, and fatty acid metabolism. In: Burtis CA, Ashwood ER, Bruns DE (eds). Tietz Textbook of Clinical Chemistry and Molecular Diagnostics, 4th edn. Elsevier Saunders: St. Louis, MO, 2005:2207-2247.

12. Seymour CA, Thomason MJ, Chalmers RA, et al. Newborn screening for inborn errors of metabolism: a systematic review. Health Technol Assess 1997; 1:i-iv, 1-95.

13. Applegarth DA, Toone JR \& Lowry RB. Incidence of inborn errors of metabolism in British Columbia, 1969-1996. Pediatrics 2000;105:e10.

14. Dietzen DJ, Rinaldo P, Whitley RJ, et al. National Academy of Clinical Biochemistry laboratory medicine practice guidelines: follow-up testing for metabolic disease identified by expanded newborn screening using tandem mass spectrometry; executive summary. Clin Chem 2009;55: $1615-1626$.
15. Rinaldo P. Organic acids. In: Blau N, Duran M, Gibson KM (eds). Laboratory Guide to the Methods in Biochemical Genetics. Springer: Berlin, Germany, 2008.

16. Rinaldo P. Organic acid analysis. In: Blau N, Duran M, Blaskovics ME (eds). Laboratory Guide to the Methods in Biochemical Genetics. Springer: Berlin, Germany, 2007:137-169.

17. Luis $\mathrm{PB}$, Ruiter JP, IJlst $\mathrm{L}$, et al. Inhibition of 3-methylcrotonyl-CoA carboxylase explains the increased excretion of 3-hydroxyisovaleric acid in valproate-treated patients. J Inherit Metab Dis 2012;35:443-449.

18. Hunter RW, Lawson C, Galitsiou E, Gifford F \& Neary JJ. Pyroglutamic acidosis in association with therapeutic paracetamol use. Clin Med (Lond) 2016;16:524-529.

19. Kumps A, Duez P \& Mardens Y. Metabolic, nutritional, iatrogenic, and artifactual sources of urinary organic acids: a comprehensive table. Clin Chem 2002;48:708-717.

20. Kumps A, Duez P, Genin J \& Mardens Y. Gas chromatography-mass spectrometry analysis of organic acids: altered quantitative response for aqueous calibrators and dilute urine specimens. Clin Chem 1999;45(8 pt 1):1297-1300

21. American College of Medical Genetics and Genomics. Newborn screening ACT sheets and confirmatory algorithms. https://www.acmg net/ACMG/Publications/ACT_Sheets_and_Confirmatory_Algorithms/NBS ACT_Sheets_and_Algorithm_Table/ACMG/Publications/ACT_Sheets_and_ Confirmatory Algorithms/NBS ACT Sheets and_Algorithms Table.aspx? hkey $=$ e2c16055-8cdc-4b22-a53b-b863622007c0. Accessed 6 July 2016.

22. Advisory Committee on Heritable Disorders in Newborns and Children. Recommended uniform screening panel. 2016. https://www.hrsa.gov/ advisorycommittees/mchbadvisory/heritabledisorders/ recommendedpanel/. Accessed 17 January 2017.

23. Wilson C, Knoll D, de Hora M, Kyle C, Glamuzina E \& Webster D. The risk of fatty acid oxidation disorders and organic acidemias in children with normal newborn screening. JIMD Rep 2017;35:53-58.

24. Hörster F, Kölker S, Loeber JG, Cornel MC, Hoffmann GF \& Burgard P. Newborn screening programmes in Europe, arguments and efforts regarding harmonisation: focus on organic acidurias. JIMD Rep 2017;32: 105-115.

25. Shennar HK, Al-Asmar D, Kaddoura A \& Al-Fahoum S. Diagnosis and clinical features of organic acidemias: a hospital-based study in a single center in Damascus, Syria. Qatar Med J 2015;2015:9.

26. Karam PE, Habbal MZ, Mikati MA, Zaatari GE, Cortas NK \& Daher RT. Diagnostic challenges of aminoacidopathies and organic acidemias in a developing country: a twelve-year experience. Clin Biochem 2013;46: 1787-1792.

27. Lim JS, Tan ES, John CM, et al. Inborn error of metabolism (IEM) screening in Singapore by electrospray ionization-tandem mass spectrometry (ESI/MS/MS): an 8 year journey from pilot to current program. $\mathrm{Mol}$ Genet Metab 2014;113:53-61.

28. Oglesbee D, Cowan TM, Pasquali M, et al. CAP/ACMG proficiency testing for biochemical genetics laboratories: a summary of performance. Genet Med 2018;20:83-90.

29. Prasad C, Salvadori MI \& Rupar CA. Severe phenotypic spectrum of mevalonate kinase deficiency with minimal mevalonic aciduria. Mol Genet Metab 2012;107:756-759.

30. Hoffmann GF, Sweetman L, Bremer HJ, et al. Facts and artefacts in mevalonic aciduria: development of a stable isotope dilution GCMS assay for mevalonic acid and its application to physiological fluids, tissue samples, prenatal diagnosis and carrier detection. Clin Chim Acta 1991;198:209-227.

31. Jones PM \& Bennett MJ. Urine organic acid analysis for inherited metabolic disease using gas chromatography-mass spectrometry. Methods Mol Biol 2010;603:423-431.

32. Duez P, Kumps A \& Mardens Y. GC-MS profiling of urinary organic acids evaluated as a quantitative method. Clin Chem 1996;42:1609-1615.

33. Woontner M \& Goodman SI. Chromatographic analysis of amino and organic acids in physiological fluids to detect inborn errors of metabolism. Curr Protoc Hum Genet 2006; Chapter 17:Unit 17.2.

34. Kawana S, Nakagawa K, Hasegawa Y, Kobayashi H \& Yamaguchi S. Improvement of sample throughput using fast gas chromatography mass-spectrometry for biochemical diagnosis of organic acid disorders. Clin Chim Acta 2008;392:34-40.

35. Shoemaker JD \& Elliott WH. Automated screening of urine samples for carbohydrates, organic and amino acids after treatment with urease. $J$ Chromatogr 1991;562:125-138. 
36. Kato S, Nakajima $Y$, Awaya $R$, et al. Pitfall in the diagnosis of fructose1,6-bisphosphatase deficiency: difficulty in detecting glycerol-3phosphate with solvent extraction in urinary GC/MS analysis. Tohoku J Exp Med 2015;237:235-239.

37. Wittmann G, Karg E, Muhl A, Bodamer OA \& Turi S. Comparison of tetrahydrofuran and ethyl acetate as extraction solvents for urinary organic acid analysis. J Inherit Metab Dis 2008;31:73-80.

38. Liu A, Kushnir MM, Roberts WL \& Pasquali M. Solid phase extraction procedure for urinary organic acid analysis by gas chromatography mass spectrometry. J Chromatogr B Analyt Technol Biomed Life Sci 2004;806: 283-287.

39. Mardens $Y$, Kumps A, Planchon C \& Wurth C. Comparison of two extraction procedures for urinary organic acids prior to gas chromatography-mass spectrometry. J Chromatogr 1992;577:341-346.

40. Westgard J \& Klee GG. Quality management. In: Burtis C, Ashwood EG (eds). Tietz Textbook of Clinical Chemistry, 3rd edn. WB Saunders: Philadelphia, PA, 1999:384-419.

41. Fowler B, Burlina A, Kozich V \& Vianey-Saban C. Quality of analytical performance in inherited metabolic disorders: the role of ERNDIM. J Inherit Metab Dis 2008;31:680-689.

42. Viau K, Ernst SL, Vanzo RJ, Botto LD, Pasquali M \& Longo N. Glutaric acidemia type 1: outcomes before and after expanded newborn screening. Mol Genet Metab 2012;106:430-438.

43. Frauendienst-Egger G \& Trefz F. Metagene: Metabolic \& Genetic Information Center. http://metagene.de/index.html. Accessed 8 July 2016.

44. Ferri L, Funghini S, Fioravanti A, et al. Aminoacylase I deficiency due to ACY1 mRNA exon skipping. Clin Genet 2014;86:367-372.

45. Lee HC, Lai CK, Yau KC, et al. Non-invasive urinary screening for aromatic L-amino acid decarboxylase deficiency in high-prevalence areas: a pilot study. Clin Chim Acta 2012;413:126-130.

46. Abdenur JE, Abeling N, Specola N, et al. Aromatic L-aminoacid decarboxylase deficiency: unusual neonatal presentation and additional findings in organic acid analysis. Mol Genet Metab 2006;87: 48-53.

47. Sloan JL, Johnston JJ, Manoli I, et al. Exome sequencing identifies ACSF3 as a cause of combined malonic and methylmalonic aciduria. Nat Genet 2011;43:883-886.

48. Peters H, Ferdinandusse S, Ruiter JP, Wanders RJ, Boneh A \& Pitt J. Metabolite studies in HIBCH and ECHS1 defects: implications for screening. Mol Genet Metab 2015;115:168-173.

49. Pitt JJ, Peters $H$, Boneh $A$, et al. Mitochondrial 3-hydroxy-3methylglutaryl-CoA synthase deficiency: urinary organic acid profiles and expanded spectrum of mutations. J Inherit Metab Dis 2015:38: 459-466.

50. Conboy E, Vairo F, Schultz M, et al. Mitochondrial 3-hydroxy-3methylglutaryl-CoA synthase deficiency: unique presenting laboratory values and a review of biochemical and clinical features. JIMD Rep; e-pub ahead of print 14 October 2017.

51. Waters PJ, Thuriot F, Clarke JT, et al. Methylmalonyl-coA epimerase deficiency: a new case, with an acute metabolic presentation and an intronic splicing mutation in the MCEE gene. Mol Genet Metab Rep 2016;9:19-24.

52. Smith A, McBride S, Marcadier JL, et al. Severe neonatal presentation of mitochondrial citrate carrier (SLC25A1) deficiency. JIMD Rep 2016;30: 73-79.

53. Santra S, Cameron JM, Shyr C, et al. Cytosolic phosphoenolpyruvate carboxykinase deficiency presenting with acute liver failure following gastroenteritis. Mol Genet Metab 2016;118:21-27.

54. Bosch AM, Abeling NG, Ijlst L, et al. Brown-Vialetto-Van Laere and Fazio Londe syndrome is associated with a riboflavin transporter defect mimicking mild MADD: a new inborn error of metabolism with potential treatment. J Inherit Metab Dis 2011;34:159-164.

55. Alfardan J, Mohsen AW, Copeland S, et al. Characterization of new ACADSB gene sequence mutations and clinical implications in patients with 2-methylbutyrylglycinuria identified by newborn screening. Mol Genet Metab 2010;100:333-338.

56. Korman SH, Andresen BS, Zeharia A, Gutman A, Boneh A \& Pitt JJ. 2-ethylhydracrylic aciduria in short/branched-chain acyl-CoA dehydrogenase deficiency: application to diagnosis and implications for the R-pathway of isoleucine oxidation. Clin Chem 2005;51:610-617.

57. El-Hattab AW \& Scaglia F. SUCLA2-related mitochondrial DNA depletion syndrome, encephalomyopathic form with methylmalonic aciduria. In: Pagon RA, Adam MP, Ardinger HH, et al. (eds). GeneReviews. University of Washington: Seattle, WA, 1993.

58. Valayannopoulos V, Haudry C, Serre V, et al. New SUCLG1 patients expanding the phenotypic spectrum of this rare cause of mild methylmalonic aciduria. Mitochondrion 2010;10:335-341.

59. Gibson KM, Goodman SI, Frerman FE \& Glasgow AM. Succinic semialdehyde dehydrogenase deficiency associated with combined 4-hydroxybutyric and dicarboxylic acidurias: potential for clinical misdiagnosis based on urinary organic acid profiling. I Pediatr 1989;114(4 pt 1):607-610.

60. Christensen M, Duno M, Lund AM, Skovby F \& Christensen $E$. Xanthurenic aciduria due to a mutation in KYNU encoding kynureninase. J Inherit Metab Dis 2007;30:248-255. 